\title{
Endoscopic Ultrasound Fine-Needle Aspiration versus Fine-Needle Biopsy for Lymph Node Diagnosis: A Large Multicenter Comparative Analysis
}

\author{
Diogo Turiani Hourneaux de Moura ${ }^{1,2,3}$, Thomas R. McCarty ${ }^{1,2}$, Pichamol Jirapinyo ${ }^{1,2}$, Igor Braga Ribeiro, \\ Galileu Ferreira Ayala Farias ${ }^{3}$, Marvin Ryou ${ }^{1,2}$, Linda S. Lee ${ }^{1,2}$ and Christopher C. Thompson ${ }^{1,2}$ \\ ${ }^{1}$ Division of Gastroenterology, Hepatology and Endoscopy, Brigham and Women's Hospital, Boston MA, USA, ${ }^{2}$ Harvard Medical School, \\ Boston, MA, USA, ${ }^{3}$ Department of Gastroenterology, Hospital das Clínicas da Faculdade de Medicina da Universidade de São Paulo, São \\ Paulo, Brazil
}

Background/Aims: Endoscopic ultrasound fine-needle aspiration (EUS-FNA) is preferred for sampling of lymph nodes (LNs) adjacent to the gastrointestinal wall; however, fine-needle biopsy (FNB) may provide improved diagnostic outcomes. This study aimed to evaluate the comparative efficacy and safety of FNA versus FNB for LN sampling.

Methods: This was a multicenter retrospective study of prospectively collected data to evaluate outcomes of EUS-FNA and EUS-FNB for LN sampling. Characteristics analyzed included sensitivity, specificity, accuracy, the number of needle passes, diagnostic adequacy of rapid on-site evaluation (ROSE), cell-block analysis, and adverse events.

Results: A total of 209 patients underwent EUS-guided LN sampling. The mean lesion size was $16.22 \pm 8.03$ mm, with similar sensitivity and accuracy between FNA and FNB ([67.21\% vs. $75.00 \%$, respectively, $p=0.216]$ and $[78.80 \%$ vs. $83.17 \%$, respectively, $p=0.423]$ ). The specificity of FNB was better than that of FNA ( $100.00 \%$ vs. $93.62 \%, p=0.01)$. The number of passes required for diagnosis was not different. Abdominal and peri-hepatic LN location demonstrated FNB to have a higher sensitivity $(81.08 \%$ vs. $64.71 \%, p=0.031$ and $80.95 \%$ vs. $58.33 \%, p=0.023$ ) and accuracy ( $88.14 \%$ vs. $75.29 \%, p=0.053$ and $88.89 \%$ vs. $70.49 \%, p=0.038$ ), respectively. ROSE was a significant predictor for accuracy (odds ratio, 5.16; 95\% confidence interval, 1.15-23.08; $p=0.032$ ). No adverse events were reported in either cohort.

Conclusions: Both EUS-FNA and EUS-FNB are safe for the diagnosis of LNs. EUS-FNB is preferred for abdominal LN sampling. EUSFNA+ROSE was similar to EUS-FNB alone, showing better diagnosis for EUS-FNB than traditional FNA. While ROSE remained a significant predictor for accuracy, due to its poor availability in most centers, its use may be limited to cases with previous inconclusive diagnoses. Clin Endosc 2020;53:600-610

Key Words: Endoscopic ultrasound; Endoscopic ultrasound-guided tissue acquisition; Fine-needle aspiration; Fine-needle biopsy; Lymph nodes

\footnotetext{
Received: August 30, 2019 Revised: September 26, 2019

Accepted: September 26, 2019

Correspondence: Christopher C. Thompson

Division of Gastroenterology, Hepatology and Endoscopy, Brigham and Women's Hospital, 75 Francis Street, Boston, MA 02115, USA

Tel: +1-617-732-6389, Fax: +1-617-264-6342, E-mail: cthompson@hms.harvard. edu

ORCID: https://orcid.org/0000-0002-6105-5270
}

(c) This is an Open Access article distributed under the terms of the Creative Commons Attribution Non-Commercial License (http://creativecommons.org/ licenses/by-nc/3.0) which permits unrestricted non-commercial use, distribution, and reproduction in any medium, provided the original work is properly cited.

\section{INTRODUCTION}

Differentiation between benign and malignant lymph nodes (LNs) is essential for adequate clinical management, as an incorrect diagnosis may significantly affect patient prognosis. Endoscopic ultrasound (EUS) is well-suited to assess lymphadenopathy in the posterior mediastinum, celiac axis, and peri-intestinal area in patients with benign or malignant gastrointestinal or thoracic diseases, allowing real-time, high-resolution imaging with relatively low cost. ${ }^{1-3}$ In the past, EUS was used only to provide morphological characteristics 
such as size, echogenicity, architecture, shape, borders, and vascular pattern; however, EUS diagnosis of LN based on morphological characteristics alone has limited accuracy. ${ }^{4}$

While different size cutoffs have been proposed for each anatomical district, diagnosis based on size is associated with reduced accuracy. Previous literature has shown that up to $30 \%$ of LNs $<5 \mathrm{~mm}$ may be malignant, while LNs $>20 \mathrm{~mm}$ may be benign in the setting of inflammation..$^{1,5}$ Modalities to improve the diagnosis of LN such as contrast enhancement and elastography have been utilized, albeit with widely variable results. Although these innovations have demonstrated higher sensitivity rates than standard EUS imaging, these techniques do not include tissue sampling and yield unsatisfactory specificity rates. ${ }^{2}$ Therefore, these techniques may be helpful in delineating circumscribed malignant infiltration and in directing EUS-guided tissue sampling. ${ }^{6}$

To increase the diagnostic accuracy, EUS-guided sampling has now become the standard of care. In general, this procedure can be safely performed in an outpatient setting without general anesthesia. ${ }^{1,4}$ Compared to alternative techniques available for sampling mediastinal LNs, EUS-guided tissue sampling is safer and less invasive. Additionally, for abdominal LNs, a prospective study including 142 patients with non-diagnostic or non-feasible percutaneous image-guided sampling demonstrated that EUS-guided sampling was successful in $92 \%$ of patients and produced a diagnosis in $91 \%{ }^{8}$ EUS tissue acquisition is typically performed with EUS-fineneedle aspiration (EUS-FNA) providing material for cytological evaluation; however, the reported sensitivity of $88 \%$ and specificity of $96.4 \%$ remains less than ideal. ${ }^{4}$ These marginal results may, in part, be due to damage of the $\mathrm{LN}$ architecture, thereby limiting histological evaluation. ${ }^{1,4}$

To overcome the limitations of EUS-FNA, novel fine-needle biopsy (FNB) systems have been designed to provide a larger sample and preserve the tissue architecture of the tissue, potentially leading to better results. For the evaluation of LNs of an unknown origin, such as lymphoma, metastasis, mycobacterial infection, and sarcoidosis, core biopsy with preservation of the LN architecture is particularly important. ${ }^{8-10}$ Although FNB appears to be the best modality for LN tissue sampling, both procedures are currently equally recommended by the European Society of Gastrointestinal Endoscopy guidelines, due to lack of studies comparing these techniques.?

Because the etiology of lymphadenopathy can radically affect patient management, accurate understanding of and sampling with the best modality are pivotal to obtain precise diagnosis. To determine the best diagnostic method for mediastinal and abdominal LN sampling, we performed a multicenter study, including a large number of patients, to compare the diagnostic outcomes and adverse events associated with
EUS-FNA and EUS-FNB.

\section{MATERIALS AND METHODS}

This was a multicenter retrospective study conducted at five hospitals in Massachusetts, United States (Brigham and Women's Hospital, Massachusetts General Hospital, Brigham and Women's Faulkner Hospital, Newton-Wellesley Hospital, and North Shore Medical Center). The study was approved by the Institutional Review Board from Partners HealthCare. All consecutive patients, aged $\geq 18$ years, who had undergone EUS-guided tissue acquisition of LNs from January 2016 to January 2019 were identified from a shared prospective registry. Search terms included endoscopic ultrasound AND lymph node OR lymphadenopathy AND fine-needle aspiration (FNA) OR fine-needle biopsy (FNB). This database contains information on patient demographics (sex, age, and comorbidities), LN characteristics (location, size, shape, heterogeneity, and echogenicity), and procedure details (route of tissue sampling, needle size and type, number of passes, diagnostic adequacy of specimen on rapid on-site evaluation [ROSE] when available, and diagnostic adequacy on cell-block and on slide examination). Additionally, the database contains information regarding patient follow-up, including adverse events, other diagnostic methods if performed, oncological treatment, and surgery.

\section{Procedural technique}

All EUS-guided tissue sampling procedures were performed using a linear array echoendoscope (Olympus GF-UCT180; Olympus, Center Valley, PA, USA) under deep sedation with monitored anesthesia care by experienced endosonographers or by gastroenterology fellows under direct expert supervision. Several different needles were used during this study period, including $22 \mathrm{G}$ and $25 \mathrm{G}$ FNA needles (Expect; Boston Scientific Co., Natick, MA, USA or Echotip; Cook Medical, Winston-Salem, NC, USA or Beacon; Medtronic Co., Newton, MA, USA) and 20 G, 22 G, and 25 G FNB needles (Acquire; Boston Scientific, or SharkCore; Medtronic, or ProCore; Cook Medical). No predefined protocol was used in the study. In general, after lesions were identified and punctured under EUS guidance, a fanning technique was performed. Individual operator technique varied between centers, including stylet slow-pull technique and standard suction.

\section{Rapid on-site evaluations}

ROSE may be utilized to determine sample adequacy and establish a preliminary diagnosis using a rapid stain. ROSE was not uniformly available for all cases and all institutions 
included in our study. Therefore, ROSE was only utilized in more challenging cases, or those that had failed prior sampling. In cases where ROSE was performed, FNA specimens were laid onto slides and smeared for onsite preparation. FNB specimens were prepared using the touch imprint technique. Briefly, the tissue surface was slightly pressed onto the slides prior to staining to reduce the creation of crushing artifacts. All slides were prepared using both wet-fixed (placed in 96\% ethyl alcohol for Papanicolaou staining) and air-dried (in some cases stained with Diff-Quik) techniques.

\section{Fine-needle aspiration evaluation}

Samples obtained through EUS-FNA were transferred onto 3-12 slides. Smears were generated with a slight pressure to avoid crushing artifacts, followed by immediate placement in $96 \%$ ethyl alcohol in half of the samples, while the remaining were fixed in the air. When possible, part of the material was placed in formalin for cell-block preparation. Specimens were subsequently transferred to the pathology division for processing and Papanicolaou staining (i.e., slides in alcohol solution), Diff-Quick Staining (i.e., air-dried slides), and hematoxylin and eosin staining (i.e., cell-block). Following these processes, cytology was performed by experienced cytopathologists.

\section{Fine-needle biopsy evaluation}

FNB samples were fixed in buffered formalin (10\% formalin) and dehydrated before embedding in paraffin. Tissues were then sliced into a 4-6- $\mu \mathrm{m}$ sections and stained with hematoxylin and eosin. Additionally, in some cases, FNB specimens were prepared in slides using the touch imprint technique. All analyses were performed by experienced pathologists.

\section{Immunohistochemical evaluation}

Immunohistochemical (IHC) staining was performed for the differential diagnosis of neoplastic and non-neoplastic LNs when needed.

\section{Outcome measures}

The primary outcome was the diagnostic yield (sensitivity, specificity, positive predictive value (PPV), negative predictive value (NPV), positive likelihood ratio (+LR), negative likelihood ratio (-LR), and accuracy of EUS-FNA and EUS-FNB from cytological or histological analysis with and without IHC staining. Inconclusive specimen results and atypical cells were considered as non-neoplastic lesions, to not overestimate diagnostic yield. Malignant cells were considered neoplastic LNs, and benign cells were considered non-neoplastic LNs. Secondary outcomes included the proportion of adequate cellularity for ROSE evaluation, median number of needle passes, diagnostic result from histological (cell-block) and cytological (slides) analysis, and adverse events related to the procedure. Anatomopathological analysis after surgery was the golden standard method. However, because most patients did not undergo surgery due to advanced disease, patient follow-up for at least 6 months was also considered as the reference standard.

\section{Statistical analysis}

Baseline patient and procedure characteristics were summarized as mean \pm standard deviation for continuous data and as frequencies and proportions for categorical data. As diagnostic tests were performed in two independent groups of patients, a bivariate model was used to compute pooled sensitivity, specificity, and diagnostic accuracy. Two-sample $t$-tests for binomial proportions were utilized. ${ }^{11}$ Continuous data were compared using the two-sample $t$-test or Wilcoxon rank-sum test, and categorical data were compared using the chi-square or Fisher's exact test, as appropriate. ${ }^{12}$ Statistical significance was defined as $p<0.05$.

Subgroup analyses were then performed to evaluate the diagnostic yield of FNA and FNB for each location (mediastinal and abdominal) of LN. Additional analyses were also performed to identify the diagnostic yield of FNA alone, FNA with ROSE, FNB alone, and FNB with ROSE. From this data, sensitivity, specificity, PPV, NPV, +LR, $-\mathrm{LR}$, and accuracy were compared to determine whether ROSE was beneficial. To identify factors associated with diagnostic performance between FNA and FNB needle types, a multivariate logistic regression was performed with adjustment for clinically significant univariate findings as well as age, sex, needle type, needle size, and application of ROSE and cell-block. Results from regression analysis were expressed as beta-coefficient ( $\beta$ ) and odds ratio (OR). Statistical analyses were performed using the Stata 15.0 software package (Stata Co. LP, College Station, TX, USA).

\section{RESULTS}

\section{Baseline patient, lesion, needle, and sampling char- acteristics}

This was a retrospective chart review of a prospectively collected database that included a total of 209 patients who underwent EUS-guided FNA or FNB LN sampling ( $n=108$ FNA and $n=101 \mathrm{FNB}$ ). The mean age of all patients was $63.70 \pm 12.50$ years, with no significant difference between FNA and FNB cohorts $(64.42 \pm 11.77$ years vs. $62.93 \pm 13.26$ years, $p=0.333)$. Thirty-two percent of all patients were women, and the FNA 
Table 1. Characteristics of the Patients, Lesions, and Tissue Acquisition

\begin{tabular}{|c|c|c|c|c|}
\hline Results & Total & FNA & FNB & $p$-value \\
\hline \multicolumn{5}{|l|}{ Patient characteristics } \\
\hline No. of patients & 209 & 108 & 101 & \\
\hline Age in years (SD) & $63.70(12.50)$ & $64.42(11.77)$ & $62.93(13.26)$ & 0.333 \\
\hline Gender & & & & 0.024 \\
\hline No. of males (\%) & $142(67.94 \%)$ & $81(75.00 \%)$ & $61(60.40 \%)$ & \\
\hline No. of females (\%) & $67(32.06 \%)$ & $27(25.00 \%)$ & $40(39.60 \%)$ & \\
\hline Lymph node location (\%) & & & & 0.244 \\
\hline Peri-esophageal & $6(2.87 \%)$ & $3(2.78 \%)$ & $3(2.97 \%)$ & \\
\hline Peri-gastric & $15(7.17 \%)$ & $9(8.33 \%)$ & $6(5.94 \%)$ & \\
\hline Peri-duodenal & $2(0.95 \%)$ & $1(0.93 \%)$ & $1(0.99 \%)$ & \\
\hline Peri-hepatic & $127(60.76 \%)$ & $61(56.48 \%)$ & $66(65.35 \%)$ & \\
\hline Peri-pancreatic & $21(10.04 \%)$ & $9(8.33 \%)$ & $12(11.88 \%)$ & \\
\hline Celiac & $13(6.22 \%)$ & $6(5.56 \%)$ & $7(6.93 \%)$ & \\
\hline Mediastinal & $21(10.04 \%)$ & $17(15.74 \%)$ & $4(3.96 \%)$ & \\
\hline Peri-rectal & $4(1.91 \%)$ & $2(1.85 \%)$ & $2(1.98 \%)$ & \\
\hline Lesion size (mm) (SD) & $16.62(8.03)$ & $16.00(7.33)$ & $17.26(8.70)$ & 0.078 \\
\hline Diagnostic sample approach (\%) & & & & 0.64 \\
\hline Transesophageal & $54(27.55 \%)$ & $31(31.31 \%)$ & $23(23.71 \%)$ & \\
\hline Transgastric & $89(45.41 \%)$ & $44(44.44 \%)$ & $45(46.39 \%)$ & \\
\hline Tranduodenal & $49(25.00 \%)$ & $22(22.22)$ & $27(27.84 \%)$ & \\
\hline Transrectal & $4(2.04 \%)$ & $2(2.03 \%)$ & $2(2.06 \%)$ & \\
\hline Needle size (\%) & & & & 0.435 \\
\hline $20 \mathrm{G}$ & $1(0.48 \%)$ & $0(0 \%)$ & $1(1.01 \%)$ & \\
\hline $22 \mathrm{G}$ & $101(49.03 \%)$ & $50(46.73 \%)$ & $51(51.52 \%)$ & \\
\hline $25 \mathrm{G}$ & $104(50.49 \%)$ & $57(53.27 \%)$ & $47(47.47 \%)$ & \\
\hline No. of passes (SD) & $2.30(0.97)$ & $2.30(1.01)$ & $2.30(0.94)$ & 0.134 \\
\hline No. of samples with ROSE (\%) & & & & 0.825 \\
\hline Yes & $36(17.22 \%)$ & $18(16.67 \%)$ & $18(17.82 \%)$ & \\
\hline No & $173(82.78 \%)$ & $90(83.33 \%)$ & $83(82.18 \%)$ & \\
\hline Adequate sample for ROSE (\%) & & & & 0.146 \\
\hline Yes & $34(94.44 \%)$ & $16(88.89 \%)$ & $18(100 \%)$ & \\
\hline No & $2(5.56 \%)$ & $2(11.11 \%)$ & $0(0 \%)$ & \\
\hline No. of passes for ROSE adequacy (SD) & $2.83(1.00)$ & $2.78(1.00)$ & $2.89(1.02)$ & 0.744 \\
\hline No. of samples with cell block (\%) & & & & 0.053 \\
\hline Yes & $164(78.47 \%)$ & $79(73.15 \%)$ & $85(84.16 \%)$ & \\
\hline No & $45(21.53 \%)$ & $29(26.85 \%)$ & $16(15.84 \%)$ & \\
\hline Diagnostic findings with cell block (\%) & & & & 0.752 \\
\hline Benign & $101(61.58 \%)$ & $50(63.29 \%)$ & $51(60.00 \%)$ & \\
\hline Malignant & $54(32.93 \%)$ & $24(30.38 \%)$ & $30(35.29 \%)$ & \\
\hline Inconclusive & $9(5.49 \%)$ & $5(6.33 \%)$ & $4(4.71 \%)$ & \\
\hline No. of passes for cell block diagnosis (SD) & $2.33(0.98)$ & $2.42(1.00)$ & $2.26(1.00)$ & 0.31 \\
\hline
\end{tabular}

FNA, fine-needle aspiration; FNB, fine-needle biopsy; ROSE, rapid on-site evaluation; SD, standard deviation. 
group included more men than the FNB group (75.00\% vs. $60.40 \%, p=0.024)$. The most common location of LNs was peri-hepatic (65.13\%), which did not differ between the FNA and FNB cohorts ( $p=0.224)$; mediastinal LN sampling (10.77\%) was the next most common location, followed by peri-gastric LNs (7.69\%) and celiac LNs (6.67\%). The mean LN size was $16.62 \pm 8.03 \mathrm{~mm}$, with no difference between the FNA and FNB cohorts ( $16.00 \pm 7.33 \mathrm{~mm}$ vs. $17.26 \pm 8.70 \mathrm{~mm}, p=0.078)$.

The most commonly used needle sizes were $22 \mathrm{G}(49.03 \%)$ and $25 \mathrm{G}$ (50.49\%), with no significant difference between the FNA and FNB cohorts ( $p=0.435)$. The number of passes between lesions sampled with FNA and FNB was also not significantly different ( $2.30 \pm 1.01$ vs. $2.30 \pm 0.94, p=0.134)$. Application of ROSE was similar between the FNA and FNB samples $(16.67 \%$ vs. $17.82 \%, p=0.825)$. Among patients who underwent ROSE, sample adequacy and the number of passes was comparable between the cohorts (FNA $88.89 \%$ vs. FNB $100 \%, p=0.146$ and FNA $2.78 \pm 1.00$ vs. FNB $2.89 \pm 1.02, p=0.744$, respectively). Cell-blocks were performed more frequently for FNB samples ( $84.16 \%$ vs. $73.15 \%, p=0.053$ ), with no significant difference. Complete baseline characteristics and sampling results for all included patients as well as stratification by FNA or FNB are demonstrated in Table 1.

\section{Comparison of diagnostic characteristics}

The overall sensitivity, specificity, and accuracy of EUS-guided tissue sampling for LNs were 71.32\% (95\% confidence interval [CI], 62.70-78.83), 96.25\% (95\% CI, 89.43-99.22), and 80.86\% (95\% CI, 74.86-85.96), respectively. FNA and FNB showed similar sensitivity (67.21\% [95\%
CI, 54.00-78.69] vs. 75.00\% [95\% CI, 63.02-84.71], $p=0.216$ ). However, FNA resulted in a significantly lower specificity than FNB (93.62\% [95\% CI, 82.46-98.66] vs. 100.00\% [95\% CI, 89.42-100.00], $p=0.010)$. Accuracy was not different between FNA and FNB (78.80\% [95\% CI, 69.78-86.00] vs. 83.17\% [95\% CI, 74.42-89.88], $p=0.423$ ). No adverse events were reported in either FNA or FNB cohorts. Complete diagnostic test characteristics are shown in Table 2.

\section{Subgroup analyses}

Subgroup analyses with stratification by LN location (i.e., mediastinal versus abdominal [i.e., peri-gastric, peri-duodenal, peri-hepatic, peri-pancreatic, celiac, and peri-rectal]) were also performed. For mediastinal LNs, no difference between FNA and FNB was found ( $p>0.05$ ). For abdominal LNs, FNB showed significantly higher sensitivity $(81.08 \%$ [95\% CI, 64.84-92.04] vs. 64.71\% [95\% CI, 50.07-77.57], $p=0.031$ ) and specificity (100.00\% [95\% CI, 84.56-100.00] vs. 91.18\% [95\% CI, 76.32-98.14], $p=0.017)$ than FNA. Additionally, the accuracy of FNB was superior than that of FNA (88.14\% [95\% CI, 77.07-95.09] vs. 75.29\% [95\% CI, 64.75-84.01], $p=0.053$ ) (Table 3). For peri-hepatic lesions, FNB was associated with significantly higher sensitivity (58.33\% [95\% CI, 40.76-74.49] vs. $80.95 \%$ [95\% CI, 58.09-94.55], $p=0.023)$, specificity $(88.00 \%$ [95\% CI, 68.78-97.45] vs. $100.00 \%$ [95\% CI, 78.20-100.00], $p=0.032]$, and accuracy $(70.49 \%$ [95\% CI, 57.43-81.48] vs. $91.67 \%$ [95\% CI, 61.52-99.79], $p=0.038$ ) than FNA. There was no difference in the diagnostic yield between LNs in the other locations (Table 4).

Table 2. Comparison of Diagnostic Tests Results

\begin{tabular}{|c|c|c|c|c|}
\hline Results & Total & FNA & FNB & $p$-value \\
\hline \multicolumn{5}{|c|}{ Diagnostic test characteristics } \\
\hline Sensitivity & $\begin{array}{c}71.32 \% \\
(95 \% \mathrm{CI}, 62.70-78.83)\end{array}$ & $\begin{array}{c}67.21 \% \\
(95 \% \text { CI, } 54.00-78.69)\end{array}$ & $\begin{array}{c}75.00 \% \\
(95 \% \text { CI, 63.02-84.71) }\end{array}$ & 0.216 \\
\hline Specificity & $\begin{array}{c}96.25 \% \\
(95 \% \mathrm{CI}, 89.43-99.22)\end{array}$ & $\begin{array}{c}93.62 \% \\
(95 \% \mathrm{CI}, 82.46-98.66)\end{array}$ & $\begin{array}{c}100 \% \\
(95 \% \mathrm{CI}, 89.42-100.00)\end{array}$ & 0.01 \\
\hline Positive likelihood ratio & $\begin{array}{c}19.02 \\
(95 \% \mathrm{CI}, 6.23-58.03)\end{array}$ & $\begin{array}{c}10.53 \\
(95 \% \text { CI, 3.47-31.91) }\end{array}$ & N/A & N/A \\
\hline Negative likelihood ratio & $\begin{array}{c}0.30 \\
(95 \% \text { CI, } 0.23-0.39)\end{array}$ & $\begin{array}{c}0.35 \\
(95 \% \text { CI, } 0.24-0.51)\end{array}$ & $\begin{array}{c}0.25 \\
(95 \% \text { CI, } 0.17-0.38)\end{array}$ & 0.271 \\
\hline Positive predictive value & $\begin{array}{c}96.84 \% \\
(95 \% \text { CI, } 90.95-98.94)\end{array}$ & $\begin{array}{c}93.18 \% \\
(95 \% \mathrm{CI}, 81.85-97.64)\end{array}$ & $100 \%$ & 0.01 \\
\hline Negative predictive value & $\begin{array}{c}67.54 \% \\
(95 \% \text { CI, } 61.24-73.27)\end{array}$ & $\begin{array}{c}68.75 \% \\
(95 \% \text { CI, } 60.38-76.05)\end{array}$ & $\begin{array}{c}66.00 \% \\
(95 \% \text { CI, } 56.26-74.55)\end{array}$ & 0.672 \\
\hline Accuracy & $\begin{array}{c}80.86 \% \\
(95 \% \mathrm{CI}, 74.86-85.96)\end{array}$ & $\begin{array}{c}78.80 \% \\
(95 \% \text { CI, 69.78-86.00) }\end{array}$ & $\begin{array}{c}83.17 \% \\
(95 \% \text { CI, } 74.42-89.88)\end{array}$ & 0.423 \\
\hline
\end{tabular}

CI, confidence interval; FNA, fine-needle aspiration; FNB, fine-needle biopsy; N/A, not applicable. 
Table 3. Subgroup Analysis Comparing Endoscopic Ultrasound Fine-Needle Aspiration and Endoscopic Ultrasound Fine-Needle Biopsy in Mediastinal and Abdominal Lymph Nodes

\begin{tabular}{|c|c|c|c|c|}
\hline Mediastinal & Total & FNA & FNB & $p$-value \\
\hline Sensitivity & $\begin{array}{c}81.82 \% \\
(95 \% \text { CI, 48.22-97.72) }\end{array}$ & $\begin{array}{c}75.00 \% \\
(95 \% \text { CI, 34.91-96.81) }\end{array}$ & $\begin{array}{c}100 \% \\
(95 \% \text { CI, 29.24-100.00) }\end{array}$ & 0.961 \\
\hline Specificity & $\begin{array}{c}100 \% \\
(95 \% \text { CI, 69.15-100.00) }\end{array}$ & $\begin{array}{c}100 \% \\
(95 \% \mathrm{CI}, 66.37-100.00)\end{array}$ & $\begin{array}{c}100 \% \\
(95 \% \text { CI, } 2.50-100.00)\end{array}$ & 1 \\
\hline Positive likelihood ratio & $\mathrm{N} / \mathrm{A}$ & N/A & $\mathrm{N} / \mathrm{A}$ & $\mathrm{N} / \mathrm{A}$ \\
\hline Negative likelihood ratio & $\begin{array}{c}0.18 \\
(95 \% \mathrm{CI}, 0.05-0.64)\end{array}$ & $\begin{array}{c}0.25 \\
(95 \% \mathrm{CI}, 0.08-0.83)\end{array}$ & $\mathrm{N} / \mathrm{A}$ & $\mathrm{N} / \mathrm{A}$ \\
\hline Positive predictive value & $100 \%$ & $100 \%$ & $100 \%$ & 1 \\
\hline Negative predictive value & $\begin{array}{c}83.33 \% \\
(95 \% \text { CI, } 58.80-94.60)\end{array}$ & $\begin{array}{c}81.82 \% \\
(95 \% \text { CI, 57.54-93.73) }\end{array}$ & $100 \%$ & 0.972 \\
\hline Accuracy & $\begin{array}{c}90.48 \% \\
(95 \% \text { CI, 69.62-98.83) }\end{array}$ & $\begin{array}{c}88.24 \% \\
(95 \% \text { CI, 63.56-98.54) }\end{array}$ & $\begin{array}{c}100 \% \\
(95 \% \text { CI, 39.76-100.00) }\end{array}$ & 0.4816 \\
\hline Abdominal & Total & FNA & FNB & $p$-value \\
\hline Sensitivity & $\begin{array}{c}71.59 \% \\
(95 \% \text { CI, 60.98-80.70) }\end{array}$ & $\begin{array}{c}64.71 \% \\
(95 \% \text { CI, } 50.072-77.57)\end{array}$ & $\begin{array}{c}81.08 \% \\
(95 \% \text { CI, 64.84-92.04) }\end{array}$ & 0.031 \\
\hline Specificity & $\begin{array}{c}94.64 \% \\
(95 \% \text { CI, 85.13-98.88) }\end{array}$ & $\begin{array}{c}91.18 \% \\
(95 \% \text { CI, 76.32-98.14) }\end{array}$ & $\begin{array}{c}100 \% \\
(95 \% \mathrm{CI}, 84.56-100.00)\end{array}$ & 0.017 \\
\hline Positive likelihood ratio & $\begin{array}{c}13.36 \\
(95 \% \mathrm{CI}, 4.41-40.50)\end{array}$ & $\begin{array}{c}7.33 \\
(95 \% \mathrm{CI}, 2.44-22.02)\end{array}$ & $\mathrm{N} / \mathrm{A}$ & $\mathrm{N} / \mathrm{A}$ \\
\hline Negative likelihood ratio & $\begin{array}{c}0.30 \\
(95 \% \mathrm{CI}, 0.21-0.42)\end{array}$ & $\begin{array}{c}0.39 \\
(95 \% \mathrm{CI}, 0.26-0.57)\end{array}$ & $\begin{array}{c}0.19 \\
(95 \% \mathrm{CI}, 0.10-0.37)\end{array}$ & 0.83 \\
\hline Positive predictive value & $\begin{array}{c}95.45 \% \\
(95 \% \text { CI, 87.39-98.45) }\end{array}$ & $\begin{array}{c}91.67 \% \\
(95 \% \text { CI, } 78.56-97.06)\end{array}$ & $100 \%$ & 0.021 \\
\hline Negative predictive value & $\begin{array}{c}67.95 \% \\
(95 \% \text { CI, } 60.20-74.82)\end{array}$ & $\begin{array}{c}63.27 \% \\
(95 \% \mathrm{CI}, 53.93-71.70)\end{array}$ & $\begin{array}{c}75.58 \% \\
(95 \% \text { CI, } 61.73-85.96)\end{array}$ & 0.116 \\
\hline Accuracy & $\begin{array}{c}80.56 \% \\
(95 \% \text { CI, } 73.14-86.67)\end{array}$ & $\begin{array}{c}75.29 \% \\
(95 \% \text { CI, } 64.75-84.01)\end{array}$ & $\begin{array}{c}88.14 \% \\
(95 \% \text { CI, 77.07-95.09) }\end{array}$ & 0.053 \\
\hline
\end{tabular}

CI, confidence interval; FNA, fine-needle aspiration; FNB, fine-needle biopsy; N/A, not applicable.

\section{Diagnostic yield with or without rapid on-site evaluation}

A comparison between methods with or without ROSE was also performed. The use of ROSE with FNA increased sensitivity compared to FNA alone (97.67\% [95\% CI, 61.52-99.79] vs. $63.46 \%$ [95\% CI, 48.96-76.38], $p=0.004)$. When the accuracy of FNA+ROSE was compared with that of FNB alone, no statistical difference was found (94.44\% [95\% CI, 72.71-99.86] vs. 80.72 [95\% CI, 70.59-88.56], $p=0.161$ ) (Tables 5 and 6).

\section{Multivariate logistic regression}

Multivariate analysis was then performed to assess predictors for accuracy based on univariate analysis controlling for age, sex, needle type, needle size, the use of ROSE and cellblock analysis. According to this multivariate logistic regression analysis, ROSE was found to be a significant predictor for better accuracy (OR, 5.16; 95\% CI, 1.15-23.08; $p=0.032$; $\beta=1.64)$.

\section{DISCUSSION}

Since EUS-FNA has been considered standard of care, several studies have demonstrated the accuracy of this approach. Despite FNA being the first-line modality, diagnostic data shown in previous studies are variable, with accuracy ranging from $50.0 \%$ to $99.4 \%$ depending on $\mathrm{LN}$ location. ${ }^{3,13,14}$ These results may be related to the limitations of this method, such as damage of the LN architecture limiting histological evaluation. ${ }^{1,4}$ To overcome these limitations, FNB has more recently been used in several centers. Yet, to date, no studies have compared these techniques for LN sampling. To address this highly important question, we performed this first large multicenter study to compare between EUS-FNA and EUSFNB in this specific population with lymphadenopathy.

The acquisition of histological samples that yield an adequate amount of tissue suitable for IHC staining is pivotal for the personalized management of LNs, especially in cases of 
Table 4. Subgroup Analyses Comparing Endoscopic Ultrasound Fine-Needle Aspiration and Endoscopic Ultrasound Fine-Needle Biopsy in Different Locations of Abdominal Lymph Nodes

\begin{tabular}{|c|c|c|c|c|}
\hline Peri-gastric & Total & FNA & FNB & $p$-value \\
\hline Sensitivity & $\begin{array}{c}40.00 \% \\
(95 \% \text { CI, } 21.13-61.33)\end{array}$ & $\begin{array}{c}100 \% \\
(95 \% \text { CI, } 54.07-100.00)\end{array}$ & $\begin{array}{c}100 \% \\
(95 \% \mathrm{CI}, 39.76-100.00)\end{array}$ & N/A \\
\hline Specificity & $\begin{array}{c}100 \% \\
(95 \% \text { CI, } 47.82-100.00)\end{array}$ & $\begin{array}{c}100 \% \\
(95 \% \text { CI, 29.24-100.00) }\end{array}$ & $\begin{array}{c}100 \% \\
(95 \% \text { CI, } 15.81-100.00)\end{array}$ & $\mathrm{N} / \mathrm{A}$ \\
\hline Positive likelihood ratio & N/A & $\mathrm{N} / \mathrm{A}$ & $\mathrm{N} / \mathrm{A}$ & N/A \\
\hline Negative likelihood ratio & $\begin{array}{c}0.60 \\
(95 \% \mathrm{CI}, 0.44-0.83)\end{array}$ & N/A & N/A & N/A \\
\hline Positive predictive value & $100 \%$ & $100 \%$ & $100 \%$ & N/A \\
\hline Negative predictive value & $\begin{array}{c}25.00 \% \\
(95 \% \mathrm{CI}, 19.49-31.46)\end{array}$ & $100 \%$ & $100 \%$ & N/A \\
\hline Accuracy & $\begin{array}{c}50.00 \% \\
(95 \% \mathrm{CI}, 31.30-68.70)\end{array}$ & $\begin{array}{c}100 \% \\
(95 \% \text { CI, 66.37-100.00) }\end{array}$ & $\begin{array}{c}100 \% \\
(95 \% \text { CI, } 54.07-100.00)\end{array}$ & $\mathrm{N} / \mathrm{A}$ \\
\hline Peri-hepatic & Total & FNA & FNB & $p$-value \\
\hline Sensitivity & $\begin{array}{c}66.67 \% \\
(95 \% \text { CI, } 52.94-78.60)\end{array}$ & $\begin{array}{c}58.33 \% \\
(95 \% \text { CI, } 40.76-74.49)\end{array}$ & $\begin{array}{c}80.95 \% \\
(95 \% \text { CI, 58.09-94.55) }\end{array}$ & 0.023 \\
\hline Specificity & $\begin{array}{c}92.50 \% \\
(95 \% \text { CI, 79.61-98.43) }\end{array}$ & $\begin{array}{c}88.00 \% \\
(95 \% \mathrm{CI}, 68.78-97.45)\end{array}$ & $\begin{array}{c}100 \% \\
(95 \% \text { CI, } 78.20-100.00)\end{array}$ & 0.032 \\
\hline Positive likelihood ratio & $\begin{array}{c}8.89 \\
(95 \% \mathrm{CI}, 2.95-26.80)\end{array}$ & $\begin{array}{c}4.86 \\
(95 \% \text { CI, } 1.62-14.56)\end{array}$ & N/A & N/A \\
\hline Negative likelihood ratio & $\begin{array}{c}0.36 \\
(95 \% \mathrm{CI}, 0.25-0.53)\end{array}$ & $\begin{array}{c}0.47 \\
(95 \% \mathrm{CI}, 0.31-0.72)\end{array}$ & $\begin{array}{c}0.19 \\
(95 \% \mathrm{CI}, 0.08-0.46)\end{array}$ & 0.826 \\
\hline Positive predictive value & $\begin{array}{c}92.68 \% \\
(95 \% \mathrm{CI}, 80.77-97.45)\end{array}$ & $\begin{array}{c}87.50 \% \\
(95 \% \text { CI, 70.04-95.45) }\end{array}$ & $100 \%$ & 0.028 \\
\hline Negative predictive value & $\begin{array}{c}66.07 \% \\
(95 \% \text { CI, } 57.17-73.96)\end{array}$ & $\begin{array}{c}59.46 \% \\
(95 \% \text { CI, } 49.26-68.91)\end{array}$ & $\begin{array}{c}78.95 \% \\
(95 \% \mathrm{CI}, 60.83-90.06)\end{array}$ & 0.05 \\
\hline Accuracy & $\begin{array}{c}77.32 \% \\
(95 \% \mathrm{CI}, 67.70-85.21)\end{array}$ & $\begin{array}{c}70.49 \% \\
(95 \% \text { CI, } 57.43-81.48)\end{array}$ & $\begin{array}{c}88.89 \% \\
(95 \% \text { CI, 73.94-96.89) }\end{array}$ & 0.038 \\
\hline Peri-pancreatic & Total & FNA & FNB & $p$-value \\
\hline Sensitivity & $\begin{array}{c}76.92 \% \\
(95 \% \text { CI, 46.19-94.96) }\end{array}$ & $\begin{array}{c}60.00 \% \\
(95 \% \text { CI, } 14.66-94.73)\end{array}$ & $\begin{array}{c}87.50 \% \\
(95 \% \text { CI, } 47.35-99.68)\end{array}$ & 0.156 \\
\hline Specificity & $\begin{array}{c}100 \% \\
(95 \% \text { CI, 63.06-100.00) }\end{array}$ & $\begin{array}{c}100 \% \\
(95 \% \text { CI, 39.76-100.00) }\end{array}$ & $\begin{array}{c}100 \% \\
(95 \% \text { CI, 39.76-100.00) }\end{array}$ & $\mathrm{N} / \mathrm{A}$ \\
\hline Positive likelihood ratio & N/A & N/A & N/A & N/A \\
\hline Negative likelihood ratio & $\begin{array}{c}0.23 \\
(95 \% \mathrm{CI}, 0.09-0.62)\end{array}$ & $\begin{array}{c}0.40 \\
(95 \% \mathrm{CI}, 0.14-1.17)\end{array}$ & $\begin{array}{c}0.12 \\
(95 \% \mathrm{CI}, 0.02-0.78)\end{array}$ & 0.899 \\
\hline Positive predictive value & $100 \%$ & $100 \%$ & $100 \%$ & N/A \\
\hline Negative predictive value & $\begin{array}{c}72.73 \% \\
(95 \% \mathrm{CI}, 49.71-87.80)\end{array}$ & $\begin{array}{c}66.67 \% \\
(95 \% \text { CI, } 40.60-85.40)\end{array}$ & $\begin{array}{c}80.00 \% \\
(95 \% \text { CI, 39.00-96.16) }\end{array}$ & 0.5 \\
\hline Accuracy & $\begin{array}{c}85.71 \% \\
(95 \% \text { CI, 63.66-96.95) }\end{array}$ & $\begin{array}{c}77.78 \% \\
(95 \% \text { CI, 39.99-97.19) }\end{array}$ & $\begin{array}{c}91.67 \% \\
(95 \% \mathrm{CI}, 61.52-99.79)\end{array}$ & 0.38 \\
\hline Celiac & Total & FNA & FNB & $p$-value \\
\hline Sensitivity & $\begin{array}{c}81.82 \% \\
(95 \% \text { CI, 48.22-97.22) }\end{array}$ & $\begin{array}{c}75.00 \% \\
(95 \% \text { CI, 34.91-96.81) }\end{array}$ & $\begin{array}{c}100 \% \\
(95 \% \text { CI, 29.24-100.00) }\end{array}$ & 0.275 \\
\hline Specificity & $\begin{array}{c}100 \% \\
(95 \% \text { CI, 69.15-100.00) }\end{array}$ & $\begin{array}{c}100 \% \\
(95 \% \text { CI, 66.37-100.00) }\end{array}$ & $\begin{array}{c}100 \% \\
(95 \% \text { CI, } 2.50-100.00)\end{array}$ & $\mathrm{N} / \mathrm{A}$ \\
\hline Positive likelihood ratio & N/A & $\mathrm{N} / \mathrm{A}$ & $\mathrm{N} / \mathrm{A}$ & $\mathrm{N} / \mathrm{A}$ \\
\hline
\end{tabular}


Table 4. Continued

\begin{tabular}{lcccc}
\hline Celiac & Total & FNA & FNB & N-value \\
\hline Negative likelihood ratio & 0.18 & 0.25 & N/A & N/A \\
Positive predictive value & $(95 \%$ CI, $0.05-0.64)$ & $(95 \%$ CI, $0.08-0.83)$ & $100 \%$ & 0.368 \\
Negative predictive value & $100 \%$ & $100 \%$ & $100 \%$ & 0.482 \\
Accuracy & $83.33 \%$ & $81.83 \%$ & $100 \%$ & $(95 \%$ CI, $57.54-93.73)$ \\
& $(95 \%$ CI, $58.80-94.60)$ & $88.24 \%$ & CI, 39.76-100.00) \\
\hline
\end{tabular}

CI, confidence interval; FNA, fine-needle aspiration; FNB, fine-needle biopsy; N/A, not applicable.

Table 5. Comparison between Methods with and without Rapid on-Site Evaluation

\begin{tabular}{|c|c|c|c|c|}
\hline & FNA alone & FNA with ROSE & FNB alone & FNB with ROSE \\
\hline Sensitivity & $\begin{array}{c}63.46 \% \\
(95 \% \text { CI, } 48.96-76.38)\end{array}$ & $\begin{array}{c}97.67 \% \\
(95 \% \text { CI, 61.52-99.79) }\end{array}$ & $\begin{array}{c}69.23 \% \\
(95 \% \text { CI, } 54.90-81.28)\end{array}$ & $\begin{array}{c}77.22 \% \\
(95 \% \text { CI, 66.40-85.90) }\end{array}$ \\
\hline Specificity & $\begin{array}{c}92.68 \% \\
(95 \% \text { CI, 80.08-98.46) }\end{array}$ & $\begin{array}{c}100 \% \\
(95 \% \text { CI, } 54.07-100.00)\end{array}$ & $\begin{array}{c}100 \% \\
(95 \% \text { CI, 88.78-100.00) }\end{array}$ & $\begin{array}{c}100 \% \\
(95 \% \text { CI, 90.97-100.00) }\end{array}$ \\
\hline Positive likelihood ratio & $\begin{array}{c}8.67 \\
(95 \% \text { CI, 2.86-26.28) }\end{array}$ & N/A & N/A & N/A \\
\hline Negative likelihood ratio & $\begin{array}{c}0.39 \\
(95 \% \mathrm{CI}, 0.27-0.57)\end{array}$ & $\begin{array}{c}0.08 \\
(95 \% \mathrm{CI}, 0.01-0.54)\end{array}$ & $\begin{array}{c}0.31 \\
(95 \% \mathrm{CI}, 0.20-0.46)\end{array}$ & $\begin{array}{c}0.23 \\
(95 \% \mathrm{CI}, 0.15-0.34)\end{array}$ \\
\hline Positive predictive value & $\begin{array}{c}91.67 \% \\
(95 \% \text { CI, 78.40-97.09) }\end{array}$ & $100 \%$ & $100 \%$ & $100 \%$ \\
\hline Negative predictive value & $\begin{array}{c}66.67 \% \\
(95 \% \text { CI, 58.05-74.30) }\end{array}$ & $\begin{array}{c}85.71 \% \\
(95 \% \text { CI, } 47.88-97.51)\end{array}$ & $\begin{array}{c}65.96 \% \\
(95 \% \text { CI, } 56.31-74.44)\end{array}$ & $\begin{array}{c}68.42 \% \\
(95 \% \text { CI, } 59.08-76.48)\end{array}$ \\
\hline Accuracy & $\begin{array}{c}76.34 \% \\
(95 \% \text { CI, 66.40-84.54) }\end{array}$ & $\begin{array}{c}94.44 \% \\
(95 \% \text { CI, } 72.71-99.86)\end{array}$ & $\begin{array}{c}80.72 \% \\
(95 \% \text { CI, 70.59-88.56) }\end{array}$ & $\begin{array}{c}84.75 \\
(95 \% \text { CI, 76.97-90.70) }\end{array}$ \\
\hline
\end{tabular}

CI, confidence interval; FNA, fine-needle aspiration; FNB, fine-needle biopsy; N/A, not applicable; ROSE, rapid on-site evaluation.

Table 6. Statistical Analysis between Methods with and without Rapid on-Site Evaluation

\begin{tabular}{lcccccc}
\hline & $\begin{array}{c}\text { FNA vs. } \\
\text { FNA+ROSE } \\
(\boldsymbol{p} \text {-value })\end{array}$ & $\begin{array}{c}\text { FNA vs. FNB } \\
(\boldsymbol{p} \text {-value })\end{array}$ & $\begin{array}{c}\text { FNA vs. } \\
\text { FNB+ROSE } \\
(\boldsymbol{p} \text {-value })\end{array}$ & $\begin{array}{c}\text { FNA+ROSE vs. FNA+ROSE vs. } \\
\text { FNB } \\
(\boldsymbol{p} \text {-value })\end{array}$ & $\begin{array}{c}\text { FNB vs. } \\
\text { FNB+ROSE } \\
(\boldsymbol{p} \text {-value })\end{array}$ & $\begin{array}{c}\text { FNB+ROSE } \\
(\boldsymbol{p} \text {-value })\end{array}$ \\
\hline Sensitivity & 0.004 & 0.424 & 0.030 & 0.013 & 0.044 & 0.205 \\
Specificity & 0.238 & 0.012 & 0.003 & N/A & N/A & N/A \\
Positive likelihood ratio & N/A & N/A & N/A & N/A & N/A & N/A \\
Negative likelihood ratio & 0.837 & 0.929 & 0.835 & 0.865 & 0.897 & 0.913 \\
Positive predictive value & 0.206 & 0.007 & 0.001 & N/A & N/A & N/A \\
Negative predictive value & 0.110 & 0.922 & 0.790 & 0.101 & 0.135 & 0.715 \\
Accuracy & 0.085 & 0.485 & 0.126 & 0.161 & 0.271 & 0.454 \\
\hline
\end{tabular}

FNA, fine-needle aspiration; FNB, fine-needle biopsy; N/A, not applicable; ROSE, rapid on-site evaluation.

metastasis and lymphoma. Although EUS-FNA is accurate for the identification of neoplastic cells, it has been limiting for providing tissue for IHC staining. ${ }^{15,16}$ An alternative technique is to collect FNA samples in formalin for cell-block prepara- tion and perform subsequent histological analysis. ${ }^{6,9,17,18}$ However, this technique has demonstrated lower diagnostic rates, independent of the number of FNA passes. ${ }^{19}$ In our study, cellblock analysis was possible in $73.15 \%$ of patients after FNA 
and in $84.16 \%$ after FNB. Additionally, the number of passes required to achieve cell-block diagnosis was higher for FNA than for FNB, although this difference was not statistically significant. Despite no statistical difference, the superiority of EUS-FNB and lower number of passes required for diagnostic yield on cell-block in our study are similar to those of previous literature examining solid lesions. ${ }^{1520,21}$ Accurate diagnosis using EUS-FNA is limited due to the pauci-cellular nature of the aspirate with a significant proportion of the collected tissue being distorted or consumed during automated processing and sectioning. 15,16

In our study, EUS-FNA demonstrated an accuracy of $78.80 \%$, which was congruent with several previous reports. ${ }^{14,22}$ Despite the slight superiority of FNB (83.17\%), no statistical difference was found between FNA and FNB. Another study also evaluated FNA and FNB in the diagnosis of lymphoma; however, the tru-cut biopsy (TCB) needle, a first generation FNB, was utilized, limiting the extrapolation of these results. ${ }^{16}$ Similar to our results, no superiority was found in the TCB group. Additionally, the study illustrated the superiority of EUS-FNA when associated with flow cytometry to EUS-TCB (94.7\% vs. 73\%). Furthermore, that study concluded that EUS-guided biopsy reported a lower yield to classify Hodgkin's and low-grade lymphomas than to classify highgrade diffuse large B-cell lymphoma. ${ }^{16}$

As expected from sampling diagnostic modalities, specificity and PPV were high for both techniques, suggesting that a positive result for a neoplastic lesion is very reliable. Interestingly, our analysis showed that FNB was statistically superior to FNA in specificity (100\% vs. $93.62 \%)$. However, in both groups, NPV was low, thus a negative result cannot exclude malignant diagnosis. $+\mathrm{LR}$ measures how well a test can diagnose a lesion. The higher the $+\mathrm{LR}$, the better the test performs in identifying the precise diagnosis. In our study, because specificity for EUS-FNB was 100\%, +LR could not be calculated. -LR of a test measures how well the test performs in excluding the disease. The lower the -LR, the better the test performs in excluding a disease. In our analysis, EUS-FNB had lower -LR than FNA; however, no statistical difference was found.

Different from prior studies evaluating EUS-guided tissue LN sampling, we also performed a subgroup analysis based on LN location. ${ }^{3,16,23,24}$ In the mediastinal LN analysis, the sensitivity and accuracy of EUS-FNB appeared superior to those of EUS-FNA ( $100 \%$ and $100 \%$ vs. $75 \%$ and $88.24 \%$, respectively). However, no statistical difference was found. This may be related to the small number of patients included in this analysis. Both methods provided 100\% specificity. Our results are comparable to those of a previous study that showed no difference between EUS-FNA and the old generation FNB (TCB) in the diagnosis of thoracic lesions. ${ }^{25}$ However, in our study, which included the new generation biopsy needle, higher sensitivity and accuracy were found. For abdominal LNs, FNB showed statistically better sensitivity ( $81.08 \%$ vs. $64.71 \%)$ and specificity (100\% vs. $91.18 \%)$ than FNA. Additionally, we also analyzed abdominal LNs according to location. FNB showed significantly higher accuracy than FNA in peri-hepatic lesions ( $88.89 \%$ vs. $70.49 \%)$. Regarding peri-gastric, peri-pancreatic, and celiac LNs, no differences between the needle types were found. Similarly, in a prior study that evaluated EUS-FNA in LNs, no association between cytological adequacy and LN location was found when compared pancreatic, subcarinal, aortic pulmonary window, periesophageal and other LNs. ${ }^{24}$

Literature examining ROSE remains controversial regarding improvement in diagnostic accuracy for solid lesions. ${ }^{26,27}$ Specifically for LNs, a multicenter randomized controlled trial showed that the diagnostic yield and accuracy of EUS-FNA in mediastinal and abdominal LNs with or without ROSE were comparable. ${ }^{23}$ However, the time required to review slides was shorter and post-procedural pain was less often reported in the ROSE group. In our study, ROSE was selectively utilized, usually in cases that were more challenging or had failed prior sampling. Therefore, ROSE was performed in only $17.22 \%$ of all samples included in our analysis. All FNB samples were adequate for ROSE compared to $88.89 \%$ for FNA; however, no statistical difference was observed. In our study, ROSE improved the sensitivity of FNA samples but not of FNB samples. More importantly, and similar to a previous recent meta-analysis, we demonstrated that FNB presented similar accuracy as FNA+ROSE. ${ }^{28}$

The largest study comparing FNA and FNB in all types of solid lesions has recently shown that a lower number of passes was required for diagnostic adequacy with ROSE for FNB (1 [interquartile range, 1-2] vs. 2 [interquartile range, 1-3], $p<0.001) .{ }^{15}$ A lower number of passes may reflect a shorter procedure time, lesser risk of adverse events, and better operational efficiency for both endoscopy and cytopathology units. Superior performance of FNB over FNA was observed for both pancreatic and non-pancreatic lesions. In this study, a subgroup analysis of LN sampling was also performed. ${ }^{15}$ In contrast to our study, the number of passes required for ROSE analysis was lower for the FNB group. Interestingly, our FNA results are similar to those of that study; however, our FNB results are inferior. These inconsistent results may be explained by various factors: first, our study specifically analyzed the results of FNA and FNB in LNs, including several variables such as patient, LN, and needle characteristics. Additionally, the number of FNA was three-fold higher than that of FNB in the previous study, whereas the number of procedures was similar between the studies. ${ }^{15}$ 
In our study, we also performed a multivariate logistic regression analysis to identify predictors for better accuracy. Several potential predictors were included, such as age, needle type, needle size, use of ROSE, and cell-block. Based on this analysis, ROSE was found to be the only significant predictor for better accuracy, with an OR of 5.16. This result may be related to the superiority of FNA with ROSE versus FNA alone, as demonstrated in our subgroup analyses, and should be interpreted with caution, especially because only $17.22 \%$ of our study population underwent additional testing with ROSE. A study that evaluated EUS-FNA for mediastinal and peri-intestinal LNs also performed a multivariate regression analysis and demonstrated that echo features, the site of LN sampled, and age were associated with malignant involvement. Additionally, mediastinal LNs were 2.77 times less likely to be malignant than other LNs. ${ }^{3}$ In contrast, a large study of solid masses (not specifically evaluating LNs) showed that FNB and lesion size were associated with the need to perform only one pass to achieve onsite diagnostic adequacy and were associated with acquisition of diagnostically adequate histological specimens for offsite assessment. ${ }^{15}$ Additionally, the safety of EUS tissue sampling is well established, and a few or no adverse events have been reported in the literature. ${ }^{29}$ Severe adverse events are very rare. ${ }^{6,23,30}$ In our study, similar to several studies that compared FNA and FNB, no adverse events were reported. ${ }^{30,31}$

Currently, this is the largest study to exclusively evaluate EUS-guided LN sampling. However, we recognize some limitations to our study. This was a retrospective study with inherent limitations expected from such a design, including the possibility for selection bias, lack of randomization, and the possibility that patients are lost to follow-up. Despite the retrospective design, we believe that our results are reliable, because baseline patient and lesion characteristics were similar, except for the larger numbers of men in the FNA group, which, in our opinion, did not interfere with our results. We also included different needle sizes, which simulates real-world clinical practice, with no difference between the groups. Furthermore, a meta-analysis of randomized controlled trials did not show a significant difference between different needle sizes. ${ }^{32}$

In summary, this is the largest multicenter study to specifically compare the effectiveness and safety of EUS-FNA versus EUS-FNB for LN sampling. Both EUS-FNA and EUSFNB are safe for the diagnosis of LNs. Our results showed that EUS-FNB and EUS-FNA had similar sensitivity and accuracy in differentiating between benign and malignant LNs, with a higher specificity for FNB. The use of FNB did not reduce the number of passes required for diagnostic yield. For abdominal LNs, EUS-FNB was superior to EUS-FNA. EUSFNA with ROSE produced similar results as EUS-FNB alone; thus, the use of EUS-FNB may exclude the need for ROSE as part of routine sampling. Although ROSE in addition to traditional FNA may be deferred in favor of EUS-FNB, ROSE was still found to be a significant predictor for improved accuracy on the multivariate analysis. Despite this finding, we suggest EUS-FNB over EUS-FNA+ROSE, as ROSE is more resource-intensive and is not uniformly available at most centers. However, given its role in improving accuracy, ROSE may still be beneficial for patients with previously inconclusive EUS-FNB sampling.

\section{Conflicts of Interest}

Marvin Ryou is a consultant for Medtronic, GI Windows, EnteraSense, FujiFilm, Boston Scientific, Olympus (research grant), and Pentax. Christopher C. Thompson is a consultant for Boston Scientific, Olympus America, and Apollo Endosurgery. The other authors have no financial conflicts of interest.

\section{Author Contributions}

Conceptualization: Diogo Turiani Hourneaux de Moura, Thomas R. McCarty, Pichamol Jirapinyo, Igor Braga Ribeiro, Galileu Ferreira Ayala Farias, Marvin Ryou, Linda S. Lee, Christopher C. Thompson Data curation: DTHM

Formal analysis: DTHM, TRM, PJ

Investigation: DTHM, PJ, IBR, GFAF

Supervision: CCT

Writing-original draft: DTHM

Writing-review\&editing: TRM, PJ, IBR, GFAF, MR, LSL, CCT

ORCID

Diogo Turiani Hourneaux de Moura: https://orcid.org/0000-0002-74460355

Thomas R. McCarty: https://orcid.org/0000-0003-4517-5261

Pichamol Jirapinyo: https://orcid.org/0000-0001-5273-6851

Igor Braga Ribeiro: https://orcid.org/0000-0003-1844-8973

Galileu Ferreira Ayala Farias: https://orcid.org/0000-0003-0242-3691

Marvin Ryou: https://orcid.org/0000-0001-8120-6497

Linda S. Lee: https://orcid.org/0000-0001-9921-8920

\section{REFERENCES}

1. Lisotti A, Ricci C, Serrani M, et al. Contrast-enhanced endoscopic ultrasound for the differential diagnosis between benign and malignant lymph nodes: a meta-analysis. Endosc Int Open 2019;7:E504-E513.

2. Cui XW, Jenssen C, Saftoiu A, Ignee A, Dietrich CF. New ultrasound techniques for lymph node evaluation. World J Gastroenterol 2013;19:4850-4860.

3. Chen VK, Eloubeidi MA. Endoscopic ultrasound-guided fine needle aspiration is superior to lymph node echofeatures: a prospective evaluation of mediastinal and peri-intestinal lymphadenopathy. Am J Gastroenterol 2004;99:628-633.

4. Puli SR, Batapati Krishna Reddy J, Bechtold ML, et al. Endoscopic ultrasound: it's accuracy in evaluating mediastinal lymphadenopathy? A meta-analysis and systematic review. World J Gastroenterol 2008;14:30283037.

5. Dietrich CF, Jenssen C, Arcidiacono PG, et al. Endoscopic ultrasound: elastographic lymph node evaluation. Endosc Ultrasound 2015;4:176190. 
6. Moura DTH, de Moura EGH, Matuguma SE, et al. EUS-FNA versus ERCP for tissue diagnosis of suspect malignant biliary strictures: a prospective comparative study. Endosc Int Open 2018;6:E769-E777.

7. Dumonceau JM, Deprez PH, Jenssen C, et al. Indications, results, and clinical impact of endoscopic ultrasound (EUS)-guided sampling in gastroenterology: European Society of Gastrointestinal Endoscopy (ESGE) clinical guideline - updated January 2017. Endoscopy 2017;49:695-714.

8. Puri R, Mangla R, Eloubeidi M, Vilmann P, Thandassery R, Sud R. Diagnostic yield of EUS-guided FNA and cytology in suspected tubercular intra-abdominal lymphadenopathy. Gastrointest Endosc 2012;75:10051010.

9. De Moura DT, Chacon DA, Tanigawa R, et al. Pancreatic metastases from ocular malignant melanoma: the use of endoscopic ultrasound-guided fine-needle aspiration to establish a definitive cytologic diagnosis: a case report. J Med Case Rep 2016;10:332.

10. Nieuwoudt M, Lameris R, Corcoran C, et al. Polymerase chain reaction amplifying mycobacterial DNA from aspirates obtained by endoscopic ultrasound allows accurate diagnosis of mycobacterial disease in HIV-positive patients with abdominal lymphadenopathy. Ultrasound Med Biol 2014;40:2031-2038.

11. Pepe MS. The statistical evaluation of medical tests for classification and prediction. Oxford: Oxford University Press; 2003.

12. Campbell I. Chi-squared and Fisher-Irwin tests of two-by-two tables with small sample recommendations. Stat Med 2007;26:3661-3675.

13. Gress FG, Savides TJ, Sandler A, et al. Endoscopic ultrasonography, fine-needle aspiration biopsy guided by endoscopic ultrasonography, and computed tomography in the preoperative staging of non-small-cell lung cancer: a comparison study. Ann Intern Med 1997;127(8 Pt 1):604612.

14. Williams DB, Sahai AV, Aabakken L, et al. Endoscopic ultrasound guided fine needle aspiration biopsy: a large single centre experience. Gut 1999;44:720-726

15. Bang JY, Kirtane S, Krall K, et al. In memoriam: fine-needle aspiration, birth: fine-needle biopsy: the changing trend in endoscopic ultrasound-guided tissue acquisition. Dig Endosc 2019;31:197-202.

16. Ribeiro A, Pereira D, Escalón MP, Goodman M, Byrne GE Jr. EUS-guided biopsy for the diagnosis and classification of lymphoma. Gastrointest Endosc 2010;71:851-855.

17. De Moura DTH, Coronel M, Chacon DA, et al. Primary adenosquamous cell carcinoma of the pancreas: the use of endoscopic ultrasound guided - fine needle aspiration to establish a definitive cytologic diagnosis. Rev Gastroenterol Peru 2017;37:370-373.

18. De Moura DTH, Coronel M, Ribeiro IB, et al. The importance of endoscopic ultrasound fine-needle aspiration in the diagnosis of solid pseudopapillary tumor of the pancreas: two case reports. J Med Case Rep 2018;12:107.

19. Varadarajulu S, Bang JY, Holt BA, et al. The 25-gauge EUS-FNA needle: good for on-site but poor for off-site evaluation? Results of a randomized trial Gastrointest Endosc 2014;80:1056-1063.
20. Wang J, Zhao S, Chen Y, Jia R, Zhang X. Endoscopic ultrasound guided fine needle aspiration versus endoscopic ultrasound guided fine needle biopsy in sampling pancreatic masses: a meta-analysis. Medicine (Baltimore) 2017;96:e7452

21. Lee YN, Moon JH, Kim HK, et al. Core biopsy needle versus standard aspiration needle for endoscopic ultrasound-guided sampling of solid pancreatic masses: a randomized parallel-group study. Endoscopy 2014;46:1056-1062

22. Reed CE, Mishra G, Sahai AV, Hoffman BJ, Hawes RH. Esophageal cancer staging: improved accuracy by endoscopic ultrasound of celiac lymph nodes. Ann Thorac Surg 1999;67:319-321; discussion 322.

23. Kappelle WFW, Van Leerdam ME, Schwartz MP, et al. Rapid on-site evaluation during endoscopic ultrasound-guided fine-needle aspiration of lymph nodes does not increase diagnostic yield: a randomized, multicenter trial. Am J Gastroenterol 2018;113:677-685.

24. Cleveland P, Gill KR, Coe SG, et al. An evaluation of risk factors for inadequate cytology in EUS-guided FNA of pancreatic tumors and lymph nodes. Gastrointest Endosc 2010;71:1194-1199.

25. Storch I, Shah M, Thurer R, Donna E, Ribeiro A. Endoscopic ultrasound-guided fine-needle aspiration and Trucut biopsy in thoracic lesions: when tissue is the issue. Surg Endosc 2008;22:86-90.

26. Matynia AP, Schmidt RL, Barraza G, Layfield LJ, Siddiqui AA, Adler DG. Impact of rapid on-site evaluation on the adequacy of endoscopic-ultrasound guided fine-needle aspiration of solid pancreatic lesions: a systematic review and meta-analysis. J Gastroenterol Hepatol 2014;29:697-705.

27. Keswani RN, Krishnan K, Wani S, Keefer L, Komanduri S. Addition of endoscopic ultrasound (EUS)-guided fine needle aspiration and on-site cytology to EUS-guided fine needle biopsy increases procedure time but not diagnostic accuracy. Clin Endosc 2014;47:242-247.

28. Rodrigues-Pinto E, Jalaj S, Grimm IS, Baron TH. Impact of EUS-guided fine-needle biopsy sampling with a new core needle on the need for onsite cytopathologic assessment: a preliminary study. Gastrointest Endosc 2016;84:1040-1046

29. El H, II, Wu H, Reuss S, et al. Prospective assessment of the performance of a new fine needle biopsy device for EUS-guided sampling of solid lesions. Clin Endosc 2018;51:576-583.

30. Iglesias-Garcia J, Poley JW, Larghi A, et al. Feasibility and yield of a new EUS histology needle: results from a multicenter, pooled, cohort study. Gastrointest Endosc 2011;73:1189-1196.

31. Iwai T, Kida M, Imaizumi H, et al. Randomized crossover trial comparing EUS-guided fine-needle aspiration with EUS-guided fine-needle biopsy for gastric subepithelial tumors. Diagn Cytopathol 2018;46:228233.

32. Guedes HG, Moura DTH, Duarte RB, et al. A comparison of the efficiency of $22 \mathrm{G}$ versus $25 \mathrm{G}$ needles in EUS-FNA for solid pancreatic mass assessment: a systematic review and meta-analysis. Clinics (Sao Paulo) 2018;73:e261. 